(C) 2014 IEEE. Personal use of this material is permitted. Permission from IEEE must be obtained for all other uses, in any current or future media, including reprinting/republishing this material for advertising or promotional purposes, creating new collective works, for resale or redistribution to servers or lists, or reuse of any copyrighted component of this work in other works.

\title{
Flight Trial Demonstration of Seamless Aeronautical Networking
}

Simon Plass, Romain Hermenier (German Aerospace Center, DLR, Germany)

Oliver Lücke, Dirk Gomez Depoorter, Théophile Tordjman (Triagnosys, Germany)

Mark Chatterton (Thales Research Technologies, UK)

Massimiliano Amirfeiz, Simone Scotti (Selex ES, Italy)

Yongqiang Jay Cheng, Prashant Pillai (University of Bradford, UK)

Thomas Gräupl (University of Salzburg, Austria)

Fred Durand (SITA, France)

Ken Murphy (Airtel, Irland)

Andrew Marriott (Thales UK, UK)

Alexander Zaytsev (Monitorsoft, Russia)

\begin{abstract}
This article presents the in-flight demonstration of a new integrated aircraft communications system combining legacy and future radio technologies. This system, developed and validated under real environmental conditions during flight trials, integrates all the aeronautical service domains within a common IPv6-based aeronautical network. The flight trials were held within the framework of the European SANDRA project at Oberpfaffenhofen, Germany, in June 2013. The presented outcomes emphasize the flexibility and scalability of the developed network and demonstrate the seamless service coverage of the given architecture across different airspace domains ${ }^{1}$.
\end{abstract}

Keywords: Flight trials, Seamless Aeronautical Network, IPv6, AeroMACS, BGAN, VDL2.

\footnotetext{
${ }^{1}$ The material in this paper was presented in part at DASC 2013, Syracuse, NY, USA
} 


\section{Introduction}

Aeronautical communications are currently facing a continuous increase in capacity demand. This ceaseless request for more communication capacity is because of the constant growth in the number of passengers and, thus, aircrafts, which are expected to double by 2035 [1] and the introduction of new aeronautical communication services with high data volume demand. The latter comprise, among others, new operational safety critical services, such as 4D-trajectory and nonsafety critical services like wireless in-cabin connectivity for passengers. To cope with this high demand in communications capacity, part of the ongoing research aims at developing new concepts and technologies for future aeronautical communications (like the European SESAR Joint Undertaking program [2] and the FAA Next Generation Air Transportation System (NextGen) [3]) with a strong emphasis on the development of new link technologies, such as the terrestrial L-band Digital Aeronautical Communications System (L-DACS) link [4] and the European Space Agency (ESA) Iris program [5].

The introduction of new digital communication links is of paramount importance in the aeronautical sector as the existing Air Traffic Management (ATM) communication infrastructure already operates close to its maximum capacity [6]. Although the new systems will eventually replace the legacy communication systems, there will be a lengthy period in which an aircraft will be fitted with all of the systems for global interoperability. Hence, there is a need to integrate legacy and future data links into one large seamless aeronautical network to serve future communication demand.

The design, development, and validation of such a seamless network correspond to the focus of the European-funded research project SANDRA (Seamless Aeronautical Networking through integration of Data links Radios and Antennas) [7], which integrates different communication links (legacy and future data links) and networks (such as ATN/OSI or ATN/IPS) with all the aeronautical service domains (ATS, AOC/AAC, and APC) in a safe, high-performance, and cost effective way 
through IPv6 as the unification point. The development of the entire corresponding ground network infrastructure is also part of the SANDRA architecture. The validation of the latter was realized by performing flight trials on the airport of Oberpfaffenhofen, close to Munich, Germany [8]. This paper gives an overview of the outcomes of the first SANDRA flight trials with a strong emphasis on the seamless handovers that were carried out between legacy and future data links, namely, VDL2, BGAN, and the newly developed AeroMACS [9], thus proving the flexibility and scalability of the SANDRA network. The seamless service coverage aspect of the SANDRA architecture was demonstrated by the successful test of various applications in all aeronautical service domains.

The rest of the article is organized as follows. The SANDRA concept is introduced, followed by the details of the overall system setup and the most relevant components. The flight trials and the main results regarding handovers, network technologies, and used applications are presented.

Glossary

\begin{tabular}{|l|l|l|l|}
\hline AAC & Airline Administrative & BGAN & Broadband Global Area Network \\
& Communications & by Inmarsat \\
\hline AeroMACS & Aeronautical Mobile Airport & CLNP & Connectionless Network Protocol \\
& Communications System & & \\
\hline AOC & Airline Operations Center & IDRP & Inter-Domain Routing Protocol \\
\hline APC & Aeronautical Passenger & IPS & Internet Protocol Suite \\
& Communication & & \\
\hline ATN & Aeronautical & OSI & Open System Interconnection \\
& Telecommunication Network & & Very high frequency (VHF) \\
\hline ATS & Air Traffic Service & VDL2 & Digital Link mode 2 \\
& & &
\end{tabular}




\section{The SANDRA Concept}

The vision of SANDRA is the integration of aeronautical communications systems by using well-proved industry standards to enable a cost-efficient global provision of distributed services. SANDRA system is considered as a "system of systems" addressing four levels of integration: service, network, radio, and antenna.



Figure 1. SANDRA flight trial network architecture.

With respect to the communications network, SANDRA spans across two segments-the aircraft and ground segments, as shown in Figure 1. The aircraft segment for the flight trials contains the main functional components: the integrated router (IR); the integrated modular radio (IMR); and the antennas consisting of a satellite L-band antenna (BGAN), a VHF-band antenna, and a C-band antenna for AeroMACS. Details about the SANDRA ground network are given in the following section.

\section{System Setup}

The system setup of the SANDRA flight trials is composed of two major segments: the airborne segment and the ground infrastructure. 


\subsection{Airborne Segment}

The SANDRA airborne system was integrated in an Airbus A320 as displayed in Figure 2.

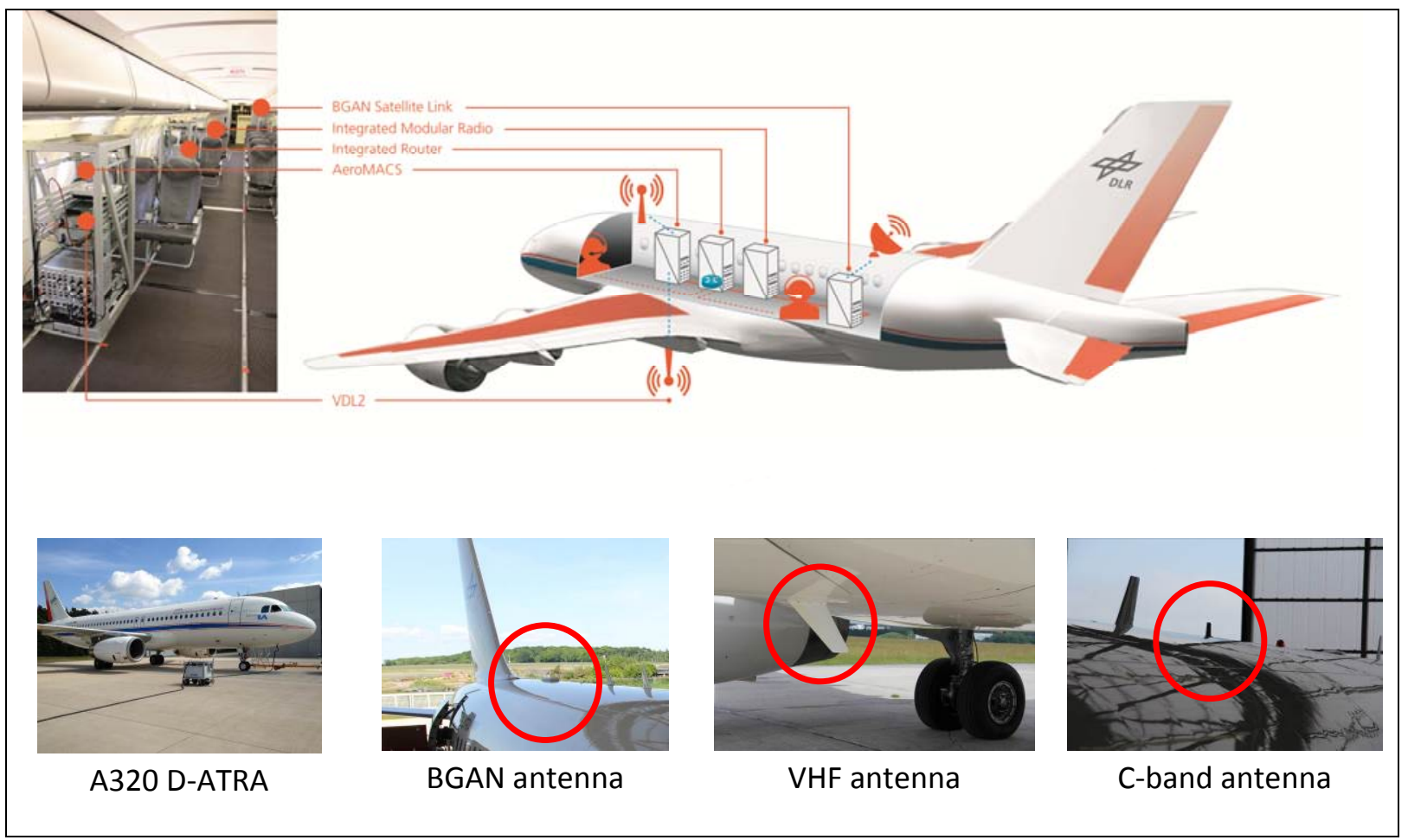

Figure 2. SANDRA airborne system installed in an A320 including the experimental antennas.

As for the data links, three different radio technologies were integrated in the aircraft: BGAN, VDL2, and AeroMACS. The aircraft was already equipped with a BGAN and a VHF antenna (used to test VDL2), which were located on top, at the rear of the fuselage, and in the middle, below the fuselage, respectively. The AeroMACS C-band antenna was especially mounted on top of the fuselage for the SANDRA flight trials. The inline figures of Figure 2 show the positions of the BGAN, VHF, and AeroMACS antennas on the fuselage of the aircraft.

To be integrated in the aircraft, the SANDRA airborne system was divided into four separate racks containing different pieces of equipment as illustrated in Figure 2. The distribution of equipment within the racks was based on the different functionalities, whereas the locations of the racks within the cabin were defined based on the positions of the antennas on the aircraft's fuselage. 
The racks were organized as follows. The first rack contained the integrated router and the connectivity to the different end-user systems. The second rack was equipped with the two integrated modular radio processing platforms, thus representing the link between the IR and the different RF equipment (one IMR used as redundancy backup, cf. IMR-PC2 in Figure A.). The third rack was fitted with the RF units for the VDL2 and AeroMACS data links. Finally, the RF components that handle the BGAN satellite link were located in the fourth rack at the rear of the cabin. To maximally reduce the antenna cable losses, the third and fourth racks were placed in the cabin right below the respective antennas.

\subsection{Ground Infrastructure}

The core part of the SANDRA ground infrastructure was located at Oberpfaffenhofen, Germany. It is composed of all the IP-based networking components, such as the access router and the home agent. The home agent includes functionalities like IPsec (IPv6) to provide authentication and integrity and the NEMO protocol [10] to guarantee mobility to the airborne terminal. Furthermore, the home agent provides global reachability accessing the mobile node via the home address during handover from/to AeroMACS to/from BGAN when attachment point changes from one access network to another. The IPsec integrates an IPv6-over-IPv4 transition mechanism, entitled NeXT [11]. The access router also provides the router advertisement messages (ICMPv6) required by NEMO on the integrated router. This message is part of the neighbor discovery protocol (NDP, RFC 4861). The SANDRA network provides connectivity not only to the different ground-end systems but also to the ATN, the Internet, and the Public Switched Telephone Network (PSTN, for passenger communication). It enables ATS (communication with air traffic control (ATC)) and AOC services (business communication of the airline) as well as APC (e.g., for Internet access and mobile telephony) and airline nonoperational services (AAC). 
For the ground infrastructure of the data links two different base stations were specifically installed for the SANDRA flight trials: a VHF ground station (VGS) and an AeroMACS base station. The latter was installed on top of a hangar building overlooking the Oberpfaffenhofen Airport. Connectivity between this base station and the SANDRA laboratory was established via a VLAN. The antenna used for the AeroMACS base station was a directional antenna $\left(90^{\circ}\right)$ with a focus on the aircraft's parking position. Furthermore, car tests were carried out at the Oberpfaffenhofen Airport to estimate the received signal level from the AeroMACS base station. A C-band antenna was mounted on the roof of a research vehicle. The signal level could be estimated on the runway, taxiing path, and parking position of the aircraft using a spectrum analyzer.

Finally, for the ATN/OSI ground infrastructure, a VGS for VDL2 was installed on the roof of the SANDRA laboratory close to the airfield, although the ATN/OSI ground-end system was located at Montreal, Canada, and connected to the SANDRA laboratory via a wide area network (WAN). The satellite connection was made over the BGAN satellite network. Further insights on the SANDRA ground infrastructure and the overall SANDRA test bed can be found in [12].

The SANDRA flight trials occurred from 24 to 26 of June 2013 at the Oberpfaffenhofen Airport (EDMO), Germany. This airport consists of one single runway. The parking position of the aircraft was in direct line-of-sight (LOS) with the AeroMACS base station and the VGS.

\section{Results of Flight Trials}

\subsection{Description of Flight Sorties}

Six sorties were made in 3 days with the D-ATRA aircraft at a rate of two flights per day. The focus of the first day was mainly to evaluate the correctness of data transmission over the air for each of the three data links. Once the links were operational, the flight trials of the second and third days aimed at validating the SANDRA concept by performing a set of scenarios that were previously 
identified. To do so, various applications ranging from ATS over AOC, AAC, and APC services were tested onboard the aircraft.

On average, each sortie lasted roughly 90 minutes including taxiing, take-off, and landing phases. The scenarios were performed onboard during the 45 minutes of cruise. For each sortie, the aircraft was flew over the Oberpfaffenhofen Airport and continued its route until the VHF connection was lost and turned around to fly back over the airport. Once out of the VHF coverage, the system seamlessly switched over to BGAN / L-Band with no loss of traffic in the scenario under test. This allowed testing the seamless functionality of the SANDRA concept.

\subsection{Seamless Aeronautical Networking Analysis}

\subsubsection{Seamless Layer 3 Handover}

Whenever a change of traffic routing policy involving two different data links occurred, a handover was performed. During the flight trials, handovers were performed between all the three link technologies in both directions (e.g., BGAN to VDL2 and VDL2 to BGAN) and also between some combinations of different quality of service contexts within the same technology (BGAN background to BGAN streaming). Additionally, the handovers were classified depending on the triggering condition. One type of handover was the "IMR triggered handover," initiated by the integrated modular radio when the aircraft was moving (or was already) out of coverage of one of the available links. The other type, the "IR triggered handover," was a handover caused by an automatic or manual change of the routing on the integrated router based on a policy (e.g., changing to a newly available and preferred link).

To test the "IMR triggered handover," an AeroMACS context was open while the aircraft was in the parking position. Once set, traffic was generated from the end systems to put some load on the link. Then, the IMR was told that the aircraft was changing from a "standing" position to "en 
route." Because AeroMACS is not available while the aircraft is cruising, the IMR initiated the procedure to open a new BGAN context and notified the IR of the upcoming change. Figure 3(a) shows the handover and how traffic is sent over the BGAN again after the handover is completed.

An "IR triggered handover" can be observed in Figure 3(b). Initially, all traffic is sent over a BGAN background context. Although this best-effort type of service is good enough for applications like browsing or e-mailing, it is not suitable for jitter-sensitive applications like voice-over-IP (VoIP). Therefore, a manual change of policy routing was performed. Instead of interrupting the traffic on the request, the traffic is routed through the new context only after this was completely established, thereby avoiding an interruption of the communication. The VolP call members did not notice any loss of communication, and in fact, no packets were lost during the handover, and only one packet suffered reordering.

It should be noted that the "IMR triggered handover" is performed when a link-in-use unexpectedly goes down. The communication is temporarily interrupted while the connection with the new link is established. "IR triggered handovers" are carried out while the link is still active, opening a context with a different link and modifying the routing only when new link is operational.

After disabling AeroMACS data link (step (1) in Figure 3(a)) the content request by the IMR from the BGAN network takes around 30sec. Now, the care-of-address can be provided towards the IR. Then, IR can initiate establishment of the IP connectivity with the ground (NeXT, NEMO, and policy routing update). Improvements in all these handover procedures can be seen here. 




Figure 3. Triggered handover performances. 


\subsubsection{Seamless Layer 2 Handover}

The IMR, which represents the data link and physical layer of the OSI stack, consists of the different radio protocol stacks (AeroMACS, VDL2, and BGAN), includes an adaptation layer called joint radio resource manager (JRRM) that is responsible for managing and controlling the underlying radios in a uniform and consistent manner, and provides a single interface to the network layer (cf. Figure A).

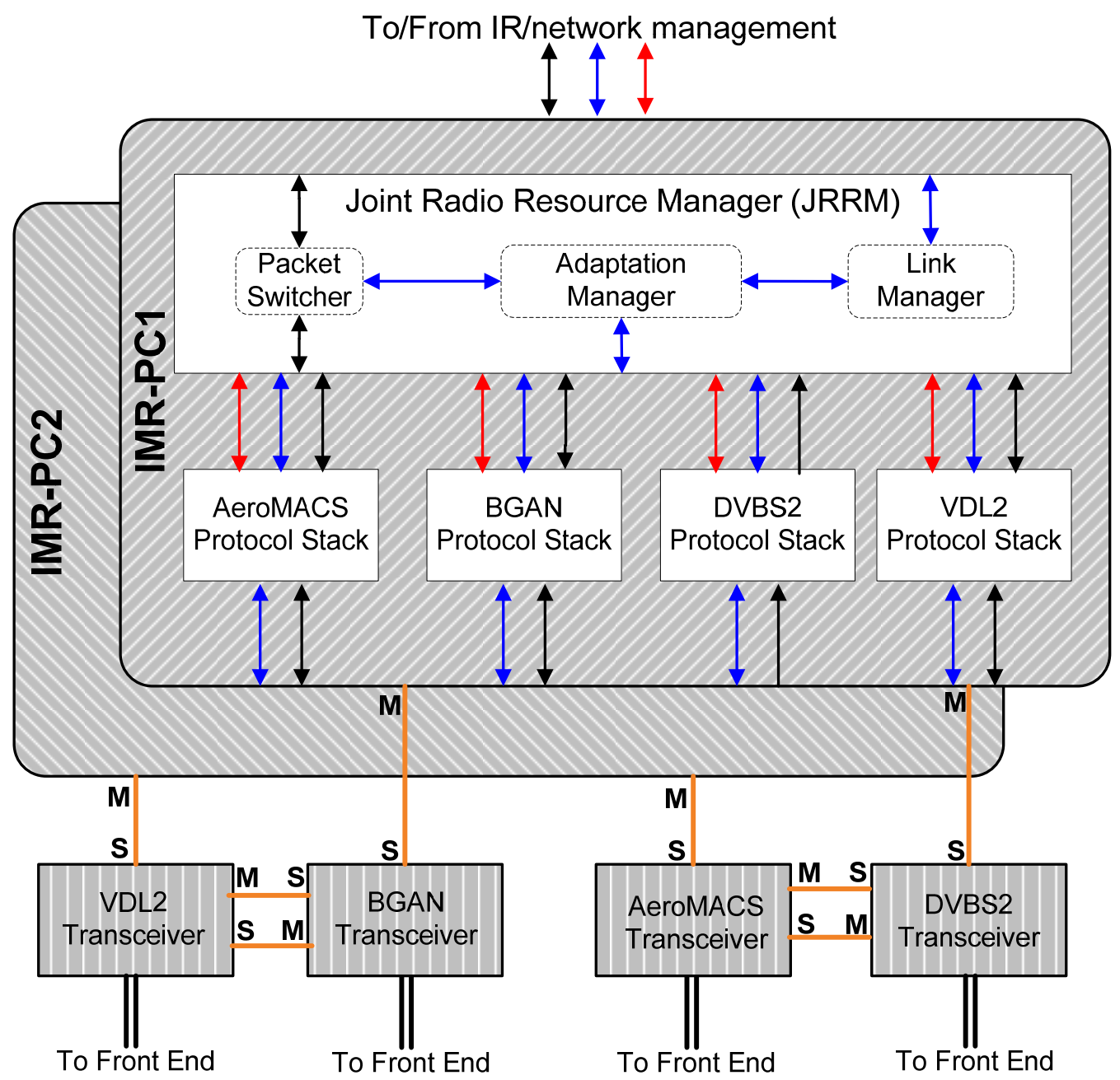

Key:

M S Optical CPRI Link (M=Master, S=Slave)

$=\mathrm{RF}$ and digital controls(e.g. antenna steering)

$\longleftrightarrow$ User Plane (User Data Flows)

$\longleftrightarrow$ Management Plane

$\longleftrightarrow$ Control Plane (Control Signaling)

Figure A. Principle architecture of the IMR. 
To increase the assurance of the IMR, there are two JRRMs running simultaneously with one on each IMR processing platform, IMR-PC1 and IMR-PC2, respectively. At any time, there is only one JRRM acting as the master and is in charge of all the processing, whereas the slave JRRM keeps on synchronizing with the master. Different time recordings for the hot swap process (when the master JRRM was terminated and the slave JRRM was swapped as the new master) could be done during the flight trials. The data-tunnel switch time indicates the time window in which data cannot be transmitted. This time varied between 170 and $184 \mathrm{~ms}$. The overall switch time is the period starting from when the slave JRRM detects a failure of the master until all submodules complete the switching process. Here, the maximum recorded time was $286 \mathrm{~ms}$, and the minimum time was 180 ms. Because of the multi-core and multi-threading programming techniques, there is not much difference between the time required for processing single or multiple data tunnels.

When the radio stacks were running on the same processing platform with the new master JRRM, it took slightly longer time for the switch process to complete because the computing resources were shared between the JRRM and radio stacks. Similarly, the data tunnel traffic load affected the switching time: the heavier the user traffic, the longer the switching time.

Figure 4 shows the time required for session establishment from randomly selected BGAN and AeroMACS sessions during the flight trial. The session establishment time is the overall time taken from the reception of the session open request until the data tunnel is completely ready for data transmission. To express the processing time required by the JRRM more precisely, the processing time in the JRRM only measures the time used within the JRRM modules by excluding the layer-two processing time of radio stacks, such as ranging, registration, or attachment time. The minimum session establishment time observed was $3.02 \mathrm{~s}$ when the satellite terminal had already been registered and attached to the network before the session open request. The maximum time observed was $22 \mathrm{~s}$ when a fresh network registration and attachment was to be done to setup and activate the packet data protocol (PDP) context for the open request. On the other hand, establishment of the AeroMACS session is quicker; it takes less than $1 \mathrm{~s}$ to complete a data 
connection with the ground station. However, processing time used for session establishment within the JRRM for BGAN and that for AeroMACS have the same order of magnitude despite the big overall difference in their end-to-end connection establishment owing to the fact that JRRM treats all the waveforms equally and in a uniform way.

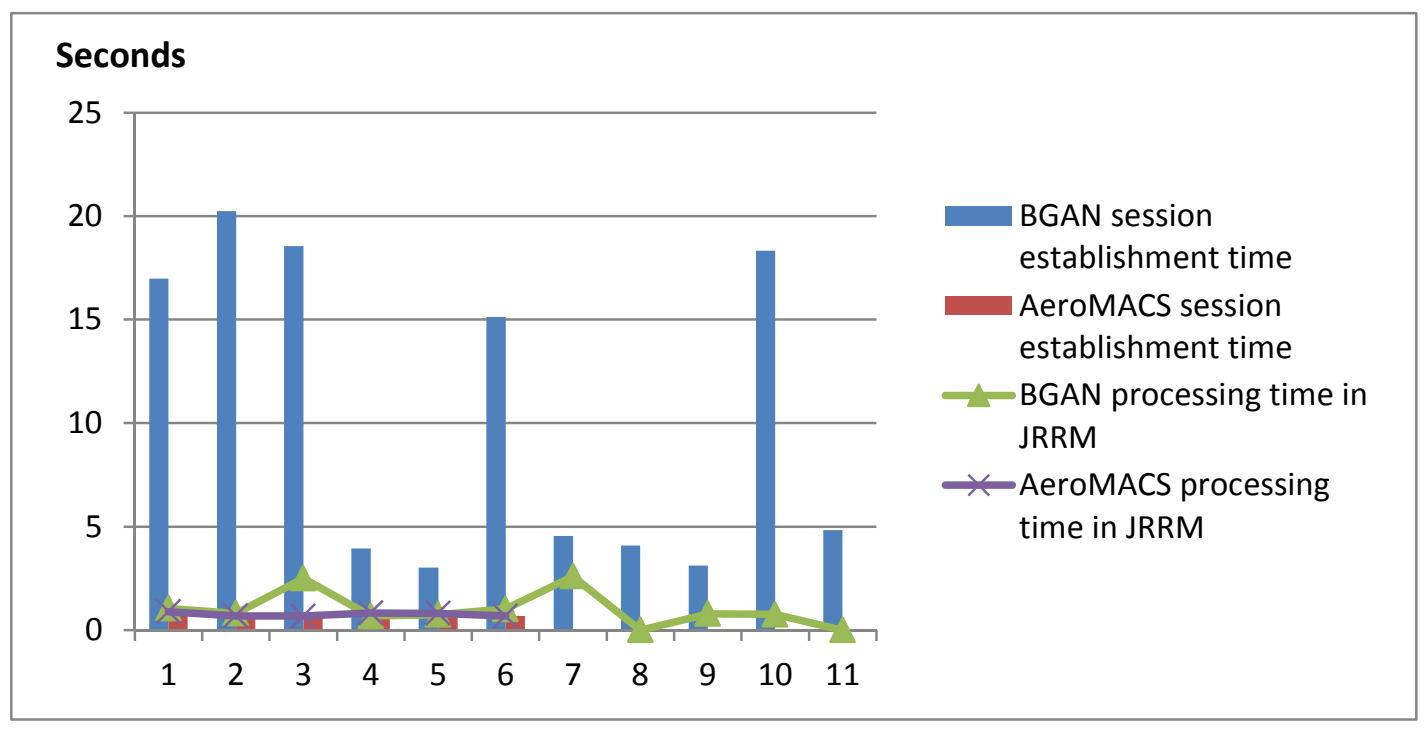

Figure 4. Session establishment time.

\subsubsection{ATN/OSI over IP-SNDCF}

The use of IP subnetwork dependent convergence function (SNDCF) enables the ATN/OSI upper layers and network (CLNP-IDRP) protocols to be conveyed over the IP protocol. This allows IPbased networks to be used for providing the underlying ATN subnetwork links between the ATNrouting entities. It was decided to experiment on the use of IP-SNDCF on the aircraft (whereas today it is only used on ground) and thus on a mobile system. The objective was to assess if the ATN/OSI CLNP packets could be conveyed over VDL2 (as done today) and over the SANDRA broadband radioIP links in a seamless way. The advantage of a mobile IP-SNDCF is that avionics and ground stations can implement a single (or multiple but standardized) SNDCF for all mobile communication technologies instead of having different interfaces for each technology, which is the case at present. 
A prototype mobile IP-SNDCF module was developed and integrated on existing ground architecture and on the aircraft, cf. Figure B. This allowed demonstrating end-to-end ATN/OSI communications over VDL2 and SANDRA mobile IP implementation over BGAN and AeroMACS [13].



Figure B. Principle architecture of ATN/OSI over mobile IP-SNDCF.

\subsection{Aeronautical Service Coverage}

Table 1 reveals the different applications that were successfully tested on ground and in the cruising phase to validate the SANDRA concept. As can be seen in Table 1, applications from all the different aeronautical service domains were tested during the flight trials, thereby emphasizing the seamless service coverage of SANDRA. As to the airborne end-system, most of the applications were 
tested using a notebook or tablet directly connected to the integrated router either via an Ethernet cable or via in-cabin wireless local access network. Their counterparts on the ground had various locations, such as the SANDRA laboratory or the different internet servers. In the following section, various applications of different service domains concerning safety relevant data, such as voice communication in the cockpit, airline operations services, and cabin communications, are highlighted. 
Table 1. List of applications tested during the SANDRA flight trials.

\begin{tabular}{|c|c|c|c|c|}
\hline Application & Domain & $\begin{array}{c}\text { Airborne } \\
\text { end-system }\end{array}$ & $\begin{array}{l}\text { Ground end- } \\
\text { system }\end{array}$ & $\begin{array}{l}\text { Ground } \\
\text { end-system } \\
\text { location }\end{array}$ \\
\hline AMBEATC10B VoIP & ATS & VoIP HW & VolP HW & SANDRA lab \\
\hline CPDLC ATN/OSI apps. & ATS & $\begin{array}{c}\text { CMU } \\
\text { Notebook }\end{array}$ & ATN ES & Montreal \\
\hline Generic CPDLC tool & ATS & Notebook & Notebook & SANDRA lab \\
\hline $\begin{array}{l}\text { Electronic flight } \\
\text { information bulletin }\end{array}$ & $A O C$ & Notebook & Server(s) & Internet \\
\hline Web chart application & $A O C$ & Notebook & Server(s) & Internet \\
\hline $\begin{array}{l}\text { Web flight planning } \\
\text { application }\end{array}$ & AOC & Notebook & Server(s) & Internet \\
\hline Electronic flight folder & AOC & Notebook & Notebook, & SANDRA lab \\
\hline Flightstrips & AAC & Notebook & Notebook & SANDRA lab \\
\hline $\begin{array}{c}\text { Generic } \\
\text { arrival/departure } \\
\text { manager }\end{array}$ & AAC & Notebook & Notebook & SANDRA lab \\
\hline Telemedicine & AAC & $\begin{array}{c}\text { Telemedicine } \\
\text { tablet }\end{array}$ & $\begin{array}{c}\text { Telemedicine } \\
\text { server }\end{array}$ & Internet \\
\hline VolP call & APC & $\begin{array}{l}\text { VolP } \\
\text { mobile }\end{array}$ & VolP Handset & Internet \\
\hline Web browser & APC & Tablet & Web server & Internet \\
\hline Email & APC & Tablet & Email Server & Internet \\
\hline Skype ${ }^{T M}$ & APC & Tablet & Skype $^{\mathrm{TM}}$ & Internet \\
\hline
\end{tabular}




\subsubsection{AMBEATC10B VoIP}

The AMBEATC10B VoIP is an experimental hardware voice-over-IP appliance based on the AMBE ATC $10 B$ vocoder circuit board. This is currently the only digital vocoder certified for air traffic control. The circuit board is integrated with a micro-controller and installed in a rack-mountable case with a push-to-talk button. The micro controller board runs a customized version of the Linux operating system that reads/writes voice samples from the vocoder board and sends/receives them over the SANDRA network using the user datagram protocol (UDP)/IPv6. Both the airborne and the ground appliances were equipped with commercially available ATC headsets.

The quality of service delivered by the SANDRA network for VoIP applications was evaluated using the AMBEATC10B VoIP appliance according to ITU recommendation P.80 "Methods for Subjective Determination of Transmission Quality." ITU P.80 defines a conversation opinion test. Two subjects engage in a set of previously arranged domain-specific conversations and rate them according to a defined scale. In addition, the subjects were interviewed to better understand the rating. In the case of the SANDRA evaluation, the conversations were constructed from the air traffic control simulation speech corpus [14]. Each conversation comprised six ATC phrases exchanged by the subjects. After the conversation, each subject was asked to provide an opinion on the transmission quality (excellent $=5, \ldots$, bad $=1$ ) and to indicate any difficulties in understanding the conversation partner (yes $=1$, no $=0$ ).

The SANDRA evaluation composed of four different speakers and 65 conversations (i.e., 390 phrases exchanged) was limited by the flight time and the number of personnel available in the aircraft. The participants were familiar with the transmission quality offered by the DSB-AM systems. The subjective rating of the voice quality should therefore be understood as relative to the established ATC voice systems.

Four of the 65 conversations were interrupted by reconfigurations of the data links. Handovers from the AeroMACS link to the satellite link were seamless and generally not noticed by 
the conversing subjects. However, the smaller round-trip delay of the AeroMACS system when compared with the satellite link was perceived. The users noticed occasional packet loss on the satellite link by missing the syllables in the conversations, but it was not perceived as a great problem.

The mean score over all the conversations was 4.33 (excellent $=5$, good $=4$ ) on the airborne side and 3.93 ( fair $=3$ ) on the groundside. The perceived lower audio quality of the ground users can be explained by the background noise in the aircraft that was included in the transmission. On the aircraft, the background noise was attenuated by the headsets, providing the airborne user with a clear reproduction of the ground signal recorded in a quiet room.

\subsubsection{CPDLC ATN/OSI Application}

For the controller-pilot data link communications (CPDLC) ATN/OSI application, the groundend system was located in Montreal, Canada. During the in-flight test of this application, a connection between the VGS on the ground and Montreal was established over the ATN/OSI ground network. The transmission of CPDLC messages was made over the VDL2 link. When the handover with BGAN (or AeroMACS) occurred, an IP connection through the SANDRA ground network was established between the airborne and the ground-end system in Montreal. The path for CPDLC messages switched thus from the ATN/OSI ground network to the IP-based SANDRA ground network. Figure 5(a) gives an example of the CPDLC request exchanged every $10 \mathrm{~s}$ during the first test on the $26^{\text {th }}$ of June. One can notice that the LACK was generally received in $1 \mathrm{~s}$ (in IP over AeroMACS).

Context management (CM)/CPDLC messages were routed seamlessly over one medium or the other, without any impact on the upper layers. During the flight tests, the IP path (BGAN or AeroMACS) was given priority, and whenever both the VDL2 and the IP paths were available at the same time, traffic was automatically routed over the IP path. When the IP path became unavailable, traffic fell back to using the VDL2 path. 


\subsubsection{Airline Operational Services}

Two applications that pertain to the operational service domain of an airliner are described in this section. These applications were integrated and tested during the flight trials. The first application aims at providing the crewmembers with the current changes that are taking place in the airspace and in airports with reference to the scope of a particular flight. The application receives basic flight details from a user and then requests for all the relevant notice to airmen (NOTAM) messages from a central server. The output data are presented in the ICAO format. The second application emulates a Web service for both basic flight planning and submission of the flight plan request to the state ATM authority. Along with the other features, this application provides a digital map with details of the current aeronautical situation, automated route selection, and numerous flight plan checks.

A dedicated route, that is, UUDD (Domodedovo, Moscow) $\rightarrow$ EDDM (Munich), was first selected for such software tests as depicted in Figure 5(b). The corresponding flight plan was successfully created and submitted. Then, a positive response (approval) was received from the ATM service. Finally, all necessary NoTAMs regarding the chosen flight were requested and received. All communications of both applications took place during the taxiing and cruise phases. Despite some loss of packets and the consequent repeated requests in the TCP/IP stack (which was not seen at the application level), the software managed to communicate with the ground successfully. 


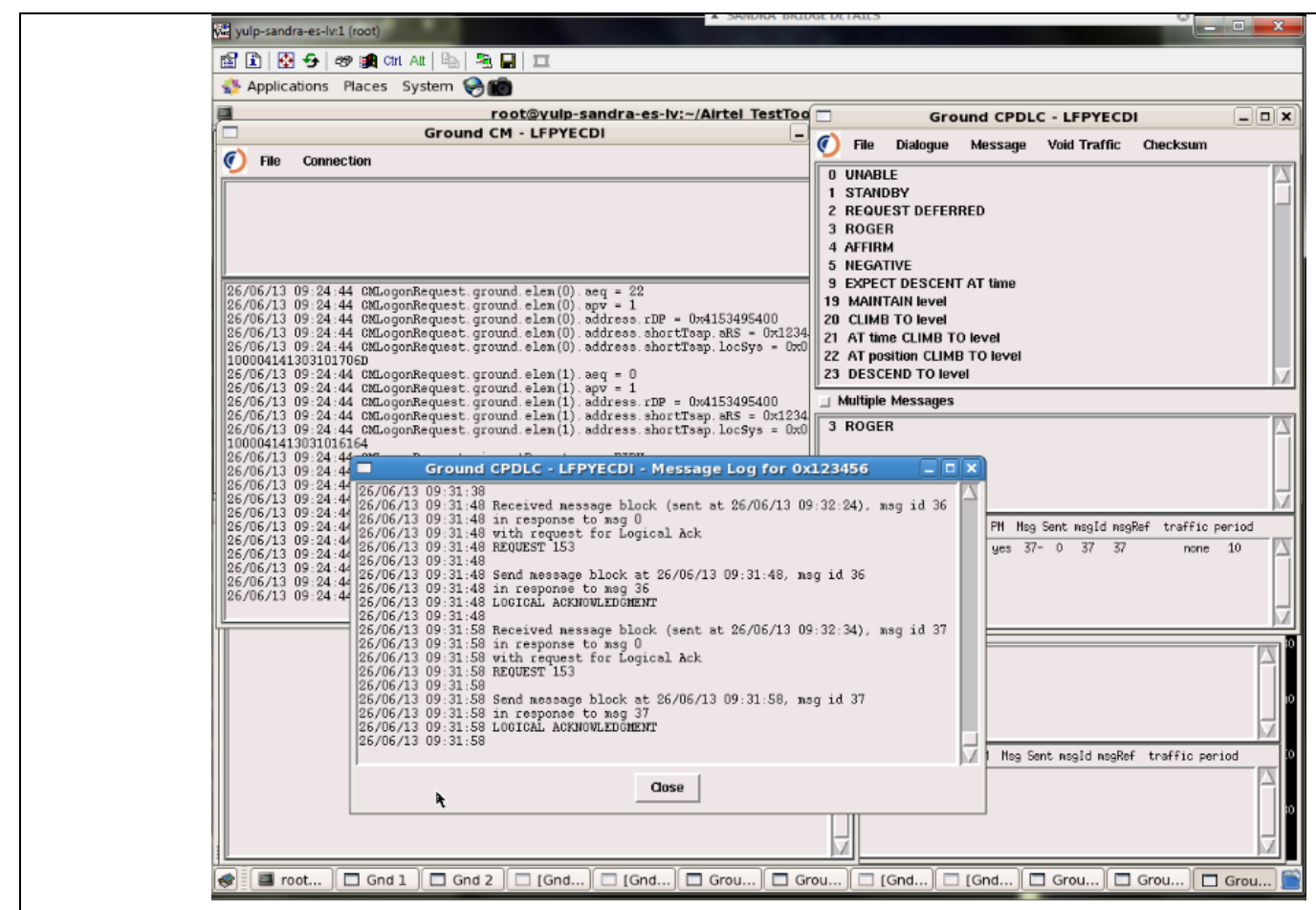

(a) CPDLC request exchanges
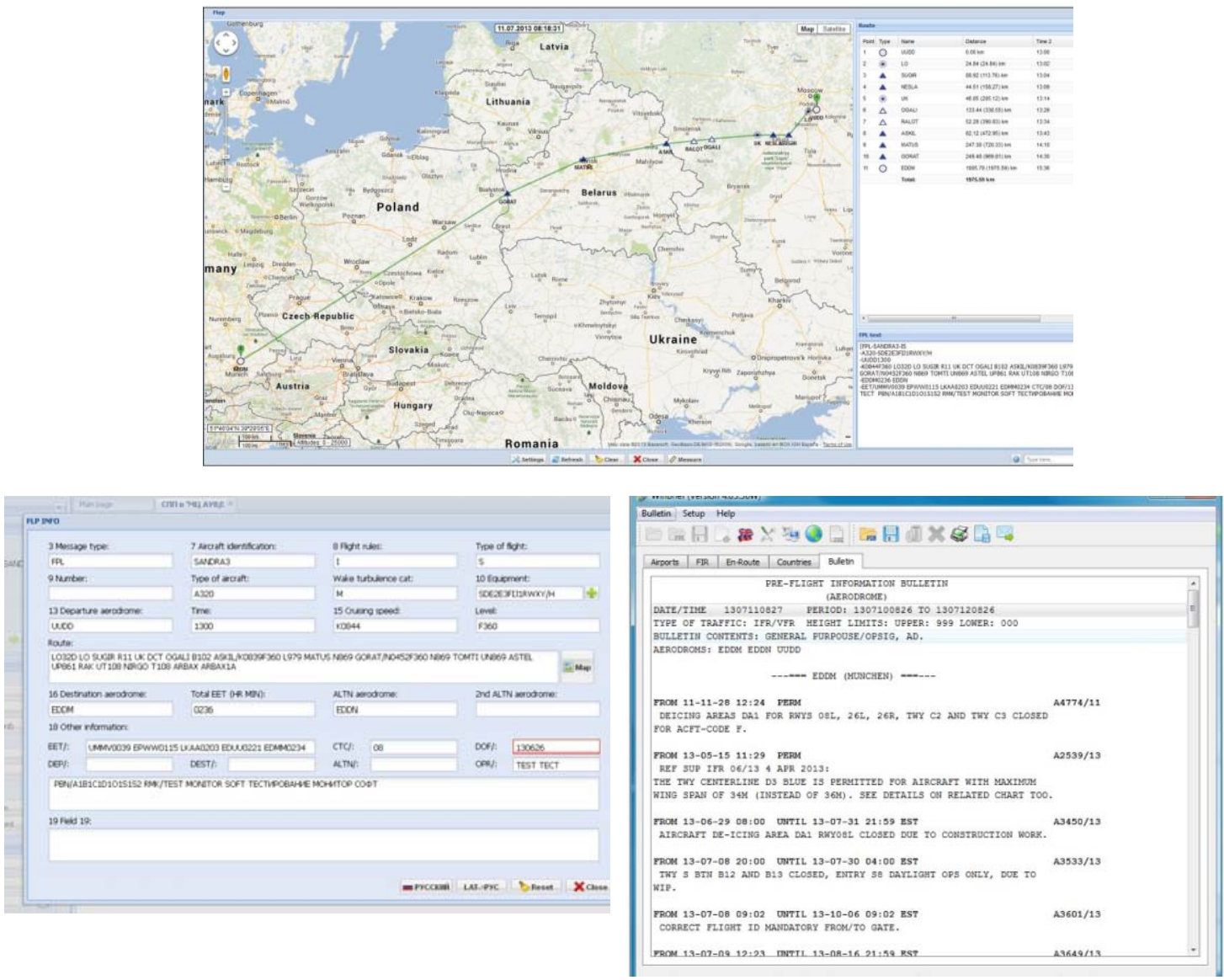

(b) Web flight planning application with ICAO FPL form submitted and NOTAMs for UUDD-EDDM at time of flight trial

Figure 5. Examples of the demonstrated applications. 


\subsubsection{Cabin Applications}

Furthermore, real-life passenger-cabin applications were tested while in flight. This involved a number of passenger scenarios including surfing the Internet and sending and receiving emails through their Internet portal. With the aid of smart phones, those in the air demonstrated social media posting and messaging as well as $\mathrm{Skype}^{\mathrm{TM}}$ video and audio calls all through the SANDRA radios. A patient monitoring unit was also demonstrated successfully for the use of the crew, whereby a crewmember and a doctor on the ground can simultaneously monitor the vital signs of a passenger while in constant audio communication through the SANDRA radio system. As part of the demonstration the unit's blood pressure monitor and pulse monitor were attached to one of the test engineers in flight. Patient monitoring units provides additional medical support to the cabin crew when it is needed most.

\subsubsection{Performance of Applications over Future Data Link}

Finally, through a closer look of the new integrated AeroMACS nonlegacy data link, it was observed that the end-to-end connectivity was affected not only by AeroMACS but also by all other networking systems (integrated router, integrated modular radio, access router, home agent, etc.). This was verified using the internet control message protocol (ICMP) pings that measured the following delays: minimum delay $=33.314 \mathrm{~ms}$, maximum delay $=265.054 \mathrm{~ms}$, average delay $=76.823$ $\mathrm{ms}$, standard deviation $=32.172 \mathrm{~ms}$, and a packet loss rate measurement of below $0.5 \%$.

Different applications successfully tested using AeroMACS connectivity are as follows:

-AMBE ATC VoIP, both over non-real-time and real-time profiles, with an average of $27 \mathrm{kbps}$ throughput during voice transmission;

-ATN/OSI over IP-SNDCF traffic, with an average throughput of $0.5 \mathrm{kbps}$ with peaks of 2.5 kbps; and 
-electronic flight folder file transfer protocol (FTP) traffic, with forward link traffic peak close to $1 \mathrm{Mbps}$, and an average traffic of below $300 \mathrm{kbps}$.

\section{Conclusions}

In this paper, the outcomes of the flight trial of a new integrated aircraft communications system were presented. Developed within the framework of the SANDRA project, this system was integrated in an Airbus A320 and tested in real flight conditions in June 2013 at the Oberpfaffenhofen Airport, Germany.

During these flight trials, the two key features of the SANDRA concept were demonstrated. On the one hand, the seamless service coverage of the SANDRA architecture across different airspace domains was shown. By keeping IPv6 as the unification point, it was proven that this system integrates a full range of aeronautical applications (ATS, AOC/AAC, and APC).

The second key feature of the SANDRA concept that was demonstrated during the flight trials was its global interoperability between legacy (VDL2 and BGAN) and future data links (AeroMACS). This was realized by performing, first, a handover on the ground between VDL2 and AeroMACS data links and, second, a handover while flying between the VDL2 and the BGAN satellite links (for both cases, handovers were performed in both directions). Transparent to the end-user, these handovers have proven the interoperable and scalable aspects of the SANDRA network, which can switch reciprocally between legacy (non-IP) and future (IP) data links. 


\section{References}

[1] EUROCONTROL: Fourth Study 'Challenges of Growth 2013, Summary Report' 2013, Available at: $\underline{\text { http://www.eurocontrol.int }}$

[2] SESAR Joint Undertaking Program: http://www.sesarju.eu/

[3] FAA, NextGen Implementation Plan, March 2010.

[4] Sajatovic, M., Haindl, B., Epple, U., Gräupl, T., Rihacek, C., Schnell, M., Fistas, N., Koch, J.-U., Kim, H.-W., \& Le-Ho, E., "Updated LDACS1 System Specification," SESAR JU, Brussels, Belgium, report EWA04-1-T2-D1, 2011.

[5] Morlet, C., Ongaro, F., Ricard, N. \& Santovincenzo, A., ESA Iris Programme: “Design options for the satellite communication sub-network of the European Air Traffic Management System," Proceedings of the 29th DASC, Salt Lake City (USA), Oct. 3-7, 2010.

[6] EUROCONTROL/FAA: "Action plan - 17: Future communication study - final conclusions and recommendations report." November 2007, Version 1.0. Available at http://www.eurocontrol.int

[7] Plass, S. "Seamless networking for aeronautical communications: One major aspect of the SANDRA concept," Aerospace and Electronic Systems Magazine, IEEE, 27(9):21-27, September 2012.

[8] Plass, S., Hermenier, R., Pulini, P., "SANDRA Flight Trials - Concept, Validation, and results," Digital Avionics Systems Conference (DASC), 2013 IEEE/AIAA 32nd, 06-10 October 2013.

[9] J. M. Budinger and E. Hall, Future Aeronautical Communications. In Tech, 2011, ch. Aeronautical Mobile Airport Communications System (AeroMACS), p. 28, Editor: S. Plass, ISBN 978-953-307$625-6$

[10] Devarapalli, V., Wakikawa, R., Petrescu, A., Thubert, P., “Network Mobility (NEMO) Basic Support Protocol," RFC 3963, January 2005. 
[11] Via, A., Fazli, E. H., Duflot, S., Riera, N., "IP Overhead Comparison in a Test-bed for Air Traffic Management Services," Proceedings of the Vehicular Technology Conference 2009 (VTC 2009), Barcelona, Spain, Apr. 26-29, 2009.

[12] Lücke, O., Depoorter, D., Tordjman, T., Kühndel, F., "The SANDRA testbed for the future aeronautical communication network," Integrated Communications, Navigation and Surveillance Conference (ICNS), 2013, 22-25 April 2013.

[13] O'Connor, F., Durand, F., "ATN/OSI over SANDRA mobile IP network: "A way to allow existing ATM solutions to leverage any broadband aircraft-ground link technologies," Integrated Communications, Navigation and Surveillance Conference (ICNS), 2013, 22-25 April 2013.

[14] Available at http://www.spsc.tugraz.at/tools/atcosim

\section{Acknowledgments}

This research was partially funded by the European Community's Seventh Framework Program (FP7/2007-2013) under Grant Agreement $n^{\circ}$ 233679. The SANDRA project is a Large Scale Integrating Project for the FP7 Topic AAT.2008.4.4.2 (Integrated approach to network-centric aircraft communications for global aircraft operations). 


\section{Biographies}

Simon Plass (M'03) received the Dipl.-Ing. and Dr.-Ing. degrees (Ph.D.) in electrical engineering from the University of Ulm, Germany, in 2003 and 2008, respectively. Since 2003, Simon has been with the Institute of Communications and Navigation of the German Aerospace Center (DLR) in Oberpfaffenhofen, Germany. He was DLR's project manager for the European research project SANDRA which integrates data links and antennas for seamless aeronautical networking. He was responsible for the overall network design, the validation and demonstration activities by test-bed and flight trials. He is co-editor of the book series Multi-Carrier Spread Spectrum and Multi-Carrier Systems \& Solutions (Wiley, 2007, 2009) and editor of the book Future Aeronautical Communications (InTech, 2011). Dr. Plass is member of the InTech Scientific Board.

Romain Hermenier is born in Le Mans, France, in 1985. He studied at the Institut National des Télécommunications (Telecom SudParis) in Evry, France from 2005 to 2007, and at the Institut Supérieur de l'aéronautique et de l'espace (Supaero), in Toulouse, France in 2008, where he specialized in the field of satellite communications. He received his Master of Engineering from Telecom SudParis in 2008. Since 2008 he is working as a scientific researcher and project manager (PMPC certified) at the German Aerospace Center (DLR) in the Institute of Communications and Navigation. He is currently involved in S-Band project activities related to the S-MIM standard.

Oliver Lücke was born in Toronto, Canada, in 1969. He received the Dipl.-Ing. degree from the Technical University of Munich (TUM), Germany, in 1997, and the Dr.-Ing. degree from the University of the Armed Forces, Munich, in 2004, both in electrical and information technologies engineering. He was a Research Assistant with the Institute for Communications Engineering of the TUM from 1997-1998 and with the Institute of Communications and Navigation of the German Aerospace Center (DLR) from 1997-2003. Since 2004 he is a Senior Engineer and Project Manager with TriaGnoSys, Wessling, Germany.

Dirk Gómez Depoorter ( $\left.M^{\prime} 14\right)$ was born in Barcelona, Spain, in 1985. He received the M.Sc. degree in aeronautical engineering from Institut Supérieur de l'Aéronautique et de l'Espace (ISAE), Toulouse, France, in 2011 and the M.Sc. degree in telecommunications engineering from Universitat Politècnica de Catalunya (UPC), Barcelona, Spain, in 2013. Since2011 he works as a research engineer at TriaGnoSys, Wessling, Germany. His research interests include network protocols and coding in the field of aeronautical and space communications.

Théophile Tordjman was born in Châtenay-Malabry, France, in 1987. He received a M.Sc. degree in computer science and automatics from the Ecole Nationale Supérieure d'ingénieurs en Informatique Automatique Mécanique Energétique et Electronique (ENSIAME), Valenciennes, France and a M.Sc. degree in communications systems engineering from the University of Valenciennes, France, both in 2011. In 2012, he obtained a DRT degree in computer science from the ENSIAME, Valenciennes, France. Since 2012 he works as a research and software engineer at TriaGnoSys, Germany.

Mark Chatterton was born in London in 1985. He received the M.Eng. degree in electronic engineering from the University of Reading in 2007. Since 2007 he has worked at Thales Research \& Technology in Reading, UK, where his interests include a wide variety of engineering topics relating to RF and telecommunications, including hardware and software development for BGAN, VDL-2 and DVB-S2 systems. Mr. Chatterton is a member of the Institute of Engineering and Technology, currently working towards Chartered Engineer status. 
Massimiliano Amirfeiz was born in Genoa, Italy, in 1970 and studied physics at the University of Genoa where he received the Dott. degree in 1996. He worked in Marconi starting from 1997 on SDH and Wavelength Division Multiplexing optical transmission systems. He has been Marconi representative at ITU-T in working groups standardizing optical interfaces. Since 2004 he joined what is now Selex ES working on aeronautical communication systems and dealing with internal and cooperative research programs. He has been project coordinator of ATENAA an FP7 project dealing with MANETs and Free Space Optics for aeronautical applications. He is SANDRA project technical coordinator since 2011, and coordinated Selex ES participation to project lab and flight test activities mainly focused on AeroMACS.

Simone Scotti was born in Genoa in 1970. He received the B.S. and M.S. degrees in electronic engineering from the University of Genoa, Italy, in 1996. In 1997 he served in the military navy. From 1998 to 2002 he has worked for Magneti Marelli company as System Test Engineer for engine power control units. Since 2002 he is employee of Selex ES, Genoa, Italy, where he worked in development of the TETRA system up to 2011 as Integration Test Engineer. Since 2012 he is involved into the development of the AeroMACS system for the SANDRA Project, as Integration Test Engineer.

Yongqiang Cheng was born in Hubei, China. He received the BEng and MEng degrees in Control Theory and Control Engineering from Tongji University, Shanghai, China in 2001 and 2004 respectively. He obtained his Ph.D. degree in 2010 from School of Engineering, Design and Technology, the University of Bradford, UK. From 2004 to 2007, he worked as a GSM/WCDMA product solution manager in the ZTE Corporation Shanghai R\&D center, where he was awarded the "Excellent Performance Staff". He is currently a postdoctoral scientific researcher in Future Ubiquitous Networking lab in School of Engineering and Informatics, the University of Bradford since 2010.

Prashant Pillai (M'03) received his B. Sc. (Hons) degree in Electronics in 2000 and M. Sc. degree in Informatics in 2002 both from University of Delhi, India and his PhD degree in the field of network security at University of Bradford, United Kingdom in 2007. He is currently working as a Senior Lecturer in the School of Engineering \& Informatics at the University of Bradford, UK. Prior to this he worked as a Lecturer from 2007 to 2013 and a Research Assistant at the University of Bradford from 2002 to 2007. He has contributed to several projects like the EU FP5 WirelessCabin Project, EU FP6 SatNEx 1 and 2 projects, Inmarsat/ESA funded Multicast BGAN Extension project. He is currently working on the EU FP7 SANDRA project and the TSB funded SINCBAC project. His main areas of work are in Mobile/wireless networks like 2G/3G, WLAN/WiMax and Bluetooth and Satellite based networks (DVB, BGAN), looking into the wireless and network control, system architecture design, protocol development and design of security architectures. He also works on various image and video processing techniques, motion detection and capturing methods, vision based robotics, eye/gaze tracking and gesture recognition systems. He also has extensive experience in designing and developing advanced embedded systems and intelligent robots. Dr Pillai is a member of IET and a fellow of the HEA.

Thomas Gräupl received the Dipl.Ing. degree in computer science from University of Salzburg, Salzburg, Austria, in 2004, the Mag. degree in mathematics from University of Salzburg, Salzburg, Austria, in 2004, and the Dr. techn. degree from University of Salzburg, Salzburg, Austria, in 2011. Since 2005 he has been a researcher at the department of computer science of the University of Salzburg, Salzburg, Austria. In 2011 he was a guest researcher at the German Aerospace Center (DLR), Oberpfaffenhofen, Germany. Since 2012 he has been a guest lecturer at the Salzburg University of Applied Sciences. He has been involved in several national and international research projects, contributed to the ICAO WG-I and WG-F working groups, and is a member of the EUROCAE WG82 standardization group. His research interests include the development and evaluation of digital aeronautical communication systems. Dr. Gräupl was the recipient of the Hans Stegbuchner 
award of the Department of Mathematics at the University of Salzburg for exceptional achievements during the curriculum.

Frederic Durand graduated from French Ecole Nationale des Telecommunications de Bretagne in 1988 (Dipl.Ing degree). Frederic is a senior engineer taking care of the definition and deployment of various data link services at SITA. He has been involved in data link since 2000. His career includes the development of avionics data buses and data link systems, at Airbus, and deployment of data link services at SITA.

Ken Murphy has over 23 years developing real-time communications software for both the telecom and aerospace industries. OSI/ATN and TCP/IP based software was developed in C/C++ using MSVC on windows and GNU GCC on Linux. Ken currently works for Airtel-ATN as a Software Architect and Subject Matter Expert (SME) for X.25, VHF Digital Link (VDL) and ACARS mobile based technology. He supports VDL based products by providing internal and external technical support and training to customers.

Andrew Marriott received the MEng Information Systems Engineering from the University of Surrey, UK in 2000. Since then is working as Systems Engineer at Thales in Crawley, UK.

Alexander Zaytsev was born in Bryansk city, Russia, in 1983. He received the B.S. and M.S. degrees in aerospace engineering from the Moscow Institute of Physics and Technology (MIPT) in 2006. From 2006 to 2010 he was working in the domain of IT security and system design. From 2010 to 2014, he was a Senior Engineer with Monitor Soft. His current research interests include airport and air traffic optimization, flight planning software development and aeronautical network technologies. 